\section{P3.031 CONDOM USE AS A FUNCTION OF NEW YOUNG ADULT RELATIONSHIP DURATION}

doi:10.1136/sextrans-2013-051184.0491

1,2 J Harezlak, ${ }^{1} \mathrm{~F} H e,{ }^{2} \mathrm{D}$ J Hensel, ${ }^{2} \mathrm{~J} \mathrm{D}$ Fortenberry. I'Indiana University Fairbanks Schoo of Public Health, Indianapolis, IN, United States; ${ }^{2}$ Indiana University School of Medicine, Indianapolis, IN, United States

Objective To find out how condom use in new relationships changes as a function of time, gender, as well as sexual and relationship satisfaction.

Method Participants in a larger study who reported at least one new partner during the 12 -week study interval $(N=115 ; 18-29$ yrs; 48\% women; 75\% African American) completed weekly STI testing and $3 \mathrm{x} /$ day electronic diary collection assessing individual and partner-specific affect, daily activities, sexual behaviour and condom use. We analysed event-level condom use percentage and subjectlevel behaviour response effects. Generalized Additive Mixed Models (GAMMs) were used to estimate condom use probability accounting for within-subject and within-nested-partners correlations via random effects.

Results The average initial condom use in the new relationships was $62 \%$ for men and $46 \%$ for women. The plotted smooth shapes of the estimated condom use probabilities fitted using GAMMs were qualitatively similar for both sexes throughout the study period. The initial high condom use percentage was followed by a sharp decline during the first 1.5 weeks to $19 \%$ for men and $14 \%$ for women. The condom use rates stabilised at around $6 \%$ after 4 weeks in a new relationship. Women who reported high levels of relationship satisfaction exhibited marginally significant negative association with condom use probability ( $p$-value $=0.055)$. Sex satisfaction was not significantly associated with condom use when both the time trends and gender were taken into account.

Conclusion Condom use declines sharply for both males and females during the early stages of new relationships. Men use condoms more frequently than women in the early and middle stages of relationships. Relationship characteristics may also influence lower levels of condom use, especially among women.

\section{P3.032 EPIDEMIOLOGY OF SEXUALLY TRANSMITTED INFECTIONS IN TVER, RUSSIA}

doi:10.1136/sextrans-2013-051184.0492

${ }^{1}$ K Koniuchova, ${ }^{1} \mathrm{M}$ Rozova, ${ }^{2} \mathrm{~A}$ Savicheva, ${ }^{3} \mathrm{E}$ Sokolovskiy, ${ }^{4} \mathrm{M}$ Domeika, ${ }^{5} \mathrm{M}$ Unemo, Eastern European Network for SexualReproductive Health. 'Avaev's Center of Specialized Medical Aid, Tver, Russian Federation; ${ }^{2}$ Ott Institute of Obstetrics and Gynecology Academy of Medical Sciences of Russian Federation, St Petersburg, Russian Federation; ${ }^{3}$ Pavlov State Medical University of St Petersburg, St Petersburg, Russian Federation, St Petersburg, Russian Federation; ${ }^{4}$ Department of Control and Prevention of Communicable Diseases, Uppsala County Council, Uppsala, Sweden; ${ }^{5}$ WHO Collaborating Centre for Gonorrhoea and other STIS, Örebro, Sweden, Örebro, Sweden

Background Tver region belongs to the Central part of Russia, its territory is $84,200 \mathrm{~km}^{2}$ with a population of $1,342,200$ inhabitants, including the city of Tver populated by 406,918 inhabitants. The aim of this paper is for the first time to internationally present the epidemiological trends of STIs in the region.

Methods Site visits and yearly epidemiological reports of the Center.

Results The peripheral laboratories are poorly equipped and therefore the diagnosis of STIs is mainly concentrated to the Center of the Specialized Medical Aid in Tver. However, the long distances for specimen transportation and lack of appropriate transport system are large obstacles for providing effective diagnosis of STIs. In 2009, vaccination against human papilloma virus (HPV) was introduced into the prevention programme against cervical cancer in Tver. The epidemiological trends of the main STIs in the Tver region are changing. A decrease in the incidence (cases per 100,000 inhabitants) of almost all STIs has been observed, namely during the years 2008 and 2012 the incidence of syphilis was 62.1 and 21.0, gonorrhoea - 42.5 and 19.2, genital chlamydial infection - 58.7 and 34.4 cases, $T$ vaginalis -120.5 and 73.6 , genital herpes -8.1 and 6.5 , and anogenital warts 21.1 and 16.6 , respectively. Since introduction in 2009, 373 girls, aged 12-13 years, have been vaccinated for HPV.

Conclusion. In Tver, Russia, the difficulties to reach the population in most need for testing is of major concern. The reported epidemiologic data is also suboptimal due to many reasons such as lack of appropriate diagnostic methods, frequent use of self-treatment (antibiotics available over the counter), and private laboratories and outpatient clinics do commonly not report STI cases to the authorities. Accordingly, it is imperative to optimise the laboratory diagnosis and epidemiological surveillance of STIs, and introduce evidence-based STI guidelines

\section{P3.033 EPIDEMIOLOGY OF VIRAL STIS IN 2000-2011 IN RUSSIAN FEDERATION}

doi:10.1136/sextrans-2013-051184.0493

E Bogdanova, L Melekhina. State Scientific Centre of Dermatovenereology g Cosmetology, Moscow, Russian Federation

Introduction and Objectives To examine epidemiology of genital herpes simplex virus and human papillomavirus (HPV) during 2000-2011 in Russian Federation.

Materials and Methods Overview of national statistical surveillance data for 2000-2011 on STI.

Results In 2011 a total of 67834 newly diagnosed cases of viral STIs were reported in Russia, which corresponds to an overall rate of 47.5 cases including genital herpes simplex virus rate of 18.3 and human papillomavirus rate 29.2 per 100,000 population.

The age-specific genital herpes simplex virus rates in 2011 were as follows: 21.8 among those aged 18 years and older, 15.7 among those aged 15-17; whereas the age-specific HPV rates were 39.0 among those aged $15-17$ versus 34.3 cases per 100,000 population among adults.

During 2000-2011 the total rates of genital herpes simplex virus remain constant (mean rate $19.8 \pm 1.4$ per 100,000 population). The total rates of HPV during the same period increase from 27.4 to 29.2 per 100,000 population with maximum reported rates of 34.7 in 2009

During 2000-2010 the mean rate of genital herpes simplex virus among adolescents aged $15-17$ was $9.5 \pm 1.2$ per 100,000 population; whereas significant increase of the rate (15.7 per 100,000 population) was observed in 2011. The mean rate of HPV among those aged 15-17 increased from 37.7 in 2000 to 39.0 in 2011 with the highest rates of 43.7 per 100,000 population reported in 2004 .

Conclusions against overall 2.6-fold decrease of total reported STI rates in 2000-2011 in Russian Federation the rates of viral STIs remain generally constant except for the 1.7 fold increase of genital herpes simplex virus rate among adolescents aged 15-17 in 2011.

\section{P3.034 GENERAL PRACTITIONERS IN THE NETHERLANDS MISS OPPORTUNITIES TO TEST FOR STI/HIV DURING STI- RELATED CONSULTATIONS}

doi:10.1136/sextrans-2013-051184.0494

II V F van den Broek, ${ }^{1,2}$ S M Trienekens, ${ }^{3} \mathrm{G}$ A Donker, ${ }^{1,4,5} \mathrm{~J}$ E A M van Bergen, ${ }^{1,6 \mathrm{M}} \mathrm{A} \mathrm{B}$ van der Sande. 'Epidemiology \& Surveillance Unit, Centre for Infectious Disease Control, National Institute of Public Health and the Environment, Bilthoven, The Netherlands, 2Department of HIV \& STI, Health Protection Services, Colindale, HPA, London, UK ${ }^{3}$ NIVEL, Dutch Sentinel Practice Network, The Netherlands Institute for Health Services research, Utrecht, The Netherlands; ${ }^{4}$ STI AIDS The Netherlands, Amsterdam, The Netherlands; ${ }^{5}$ Department of General Practice, University Medical centre, Amsterdam, The Netherlands; ${ }^{6}$ Julius Centre, University Medical Centre, Utrecht, The Netherlands 
Background Whereas the majority of STI-related consultations in the Netherlands take place in general practise (GP), national surveillance of STI predominantly uses data from STI centres, focussing at trends in high-risk groups. To also explore determinants of STI in the GP setting, an STI questionnaire was introduced in a nationwide GP-network.

Methods Since 2008, GPs of the Dutch Sentinel GP network (45 practises; 125,000 patients) are asked to complete a questionnaire for each STI-related episode, comparable to data collection in STI centres, and report laboratory results. Data included patient demographics, sexual behaviour and sex-life history.

Results Annually, for $0.4 \%$ of GP patients an STI consultation was recorded, mainly among young heterosexuals of Dutch origin, a profile comparable to STI centre visitors, though specific high-risk groups like MSM and CSW were reported less by GPs. GPs requested one or more laboratory tests in $83 \%$ of consultations; an STI was diagnosed in $34 \%$, most frequently chlamydia (21\%), condylomata $(9 \%)$ and herpes (6\%). Higher risk profiles were, depending on the STI: < 25 years old (chlamydia), MSM (gonorrhoea/syphilis), ethnic minorities (gonorrhoea), $>25$ years old (syphilis) or having symptoms (any STI). GP guidelines on multiple testing in high-risk groups (5 STI) were rarely fully adhered to, with many missed opportunities to test for HIV in patients with casual sexual contacts or originating from HIV-endemic countries.

Discussion STI consultation rates were lower than estimates based on electronic registers, probably due to underreporting. Patients who consulted a GP for STIs were comparable to persons attending STI-centres. Where STI-centres routinely test patients for chlamydia, syphilis, HIV and gonorrhoea, GPs test more selectively, resulting in higher case detection rates. This diverges from national GP guidelines and STI diagnoses may be missed. Opportunities for a more proactive role of GPs in STI and HIV testing should be explored.

\section{P3.035 PREVALENCE OF GENITAL MYCOPLASMAS AND BACTERIAL VAGINOSIS IN PREGNANT WOMEN IN GAUTENG, SOUTH AFRICA}

doi:10.1136/sextrans-2013-051184.0495

${ }^{1} \mathrm{M}$ J Redelinghuys, ${ }^{1} \mathrm{M}$ M Ehlers, ${ }^{1,2} \mathrm{~A}$ W Dreyer, ${ }^{3} \mathrm{H}$ Lombaard, ${ }^{1,2} \mathbf{M}$ M Kock 'Department of Medical Microbiology, University of Pretoria, Pretoria, South Africa; ${ }^{2}$ Tshwane Academic Division, National Health Laboratory Service, Pretoria, South Africa, ${ }^{3}$ Department of Obstetrics and Gynaecology, University of Pretoria, Pretoria, South Africa

Background Bacterial vaginosis and genital mycoplasmas are reproductive tract infections that are associated with several infections and adverse pregnancy outcomes, such as pelvic inflammatory disease, preterm birth and spontaneous abortions in affected women. Bacterial vaginosis (BV), a polymicrobial condition, is reported to be prevalent in $15 \%$ to $20 \%$ of pregnant women while mycoplasmas colonise up to about $70 \%$ of sexually active women and antenatal patients.

Methods Self-collected vaginal swabs were obtained from 221 pregnant women. Bacteria vaginosis was diagnosed using the Nugent scoring system and a multiplex PCR assay was performed to detect genital mycoplasmas. Mycoplasma hominis, M. genitalium, Ureaplasma urealyticum and U. parvum were targeted, respectively, for the $16 \mathrm{~S}$ rRNA gene, $140-\mathrm{kDa}$ adhesion protein and the multiplebanded antigen genes.

Results The prevalence of bacterial vaginosis was $17.6 \%(39 / 221)$ with a total of $23.1 \%$ (9/39) of all BV-positive cases (score 7 to 10) being HIV positive. An intermediate grading according to the Nugent system (score 4 to 6) was given to $14.9 \%$ (33/221) of samples. Ureaplasma parvum was isolated from the majority of samples with a prevalence of $72.4 \%(160 / 221)$ followed by $M$. hominis, which was present in $50.7 \%(112 / 221)$ of all samples. The prevalence of $M$. genitalium and
U. urealyticum was $14.5 \%(32 / 221)$ and $2.3 \%$ (5/221), respectively. Mycoplasma hominis was detected in $75 \%$ (27/36) of all HIV positive cases with U. parvum also present in 75\% (27/36) of these cases.

Conclusions The overall prevalence of genital mycoplasmas in antenatal patients was relatively high when compared to previous studies, while BV prevalence correlated with other reports. Ureaplasma parvum and $M$. hominis were notably present in HIV positive patients. The diagnosis of genital mycoplasmas and BV in pregnant women in HIV-prevalent South Africa is vital to allow early intervention and minimise complications, such as undesirable pregnancy outcomes.

\section{P3.036 GENITAL WARTS: DATA FROM THE ITALIAN GENERAL PRACTITIONERS}

doi:10.1136/sextrans-2013-051184.0496

'A Pasqua, ${ }^{2}$ S Costa, ${ }^{3} \mathrm{M}$ Salfa, ${ }^{4} \mathrm{~F}$ Lapi, ${ }^{3 B}$ Suligoi. ${ }^{1}$ Health Search - Italian College of General Practitioners, Florence, Italy; ${ }^{2}$ University of Bologna, Bologna, Italy; ${ }^{3}$ stituto Superiore di Sanità, Rome, Italy; ${ }^{4}$ talian College of General Practitioners, Florence, Italy

Background Worldwide, several million cases of genital warts occur each year. The objective of this study was to estimate the incidence of genital warts (GW) in Italy, in a sample of the general population aged $15-64$ years.

Methods A retrospective study was conducted in 2009 with the collaboration of 650 general practitioners (GPs), geographically representative of all Italian GPs.

Cases of GW were identified as follows: (1) individuals diagnosed with the ICD-9 code 078.11 (genital warts); or (2) individuals diagnosed with the ICD-9 code 078.10 (warts) who were prescribed podofillin/imiquimod treatment or were referred to a specialist (gynaecologist/dermatologist/urologist). Incidence was calculated using the number of persons included in the GPs lists as denominator. Individual data on age, gender, and area of residence were available.

Results The lists of the 650 GPs included 775,644 persons; their median age was 48 years, $52.7 \%$ were women. The incidence of GW was $0.44 \%$ (95\% CI $0.39-0.49)$; specifically, $0.41 \%$ among men $(95 \%$ CI $0.36-0.47)$ and $0.51 \%$ among women (95\% CI $0.43-0.57)$. The highest incidence was observed among 25-34 year-old individuals (0.64\%; $95 \%$ CI $0.52-0.78)$, for both genders. When stratifying by geographical area, the incidence was $0.40 \%$ in Northern Italy, $0.32 \%$ in Central Italy and $0.45 \%$ in Southern Italy.

Conclusions This is the first study to provide an estimate of the incidence of GW in the Italian population attending GPs. The incidence observed is similar to that reported by GPs in the UK (Desai S, 2011; Cassel JA, 2006). These results show that GPs can have a key role in preventing the spread of GW by providing early diagnosis and appropriate referral, especially among young persons, women and in persons living in Southern Italy.

\section{P3.037 HIGH PREVALENCE OF GENITAL WARTS AMONG YOUNG WOMEN IN ITALY}

doi:10.1136/sextrans-2013-051184.0497

${ }^{1} \mathrm{~L}$ Mariani, ${ }^{2} \mathrm{M}$ Salfa, ${ }^{3} \mathrm{~L}$ Timelli, ${ }^{4} \mathrm{G}$ Vittori, ${ }^{5} \mathrm{G}$ Fattorini, ${ }^{2} \mathbf{B}$ Suligoi. ${ }^{1}$ Regina Elena National Cancer Institute, HPV-Unit, Rome, Italy; 'Istituto Superiore di Sanità, Rome, Italy; ${ }^{3}$ nforma srl, Rome, Italy; ${ }^{4}$ Ospedale San Carlo di Nancy, Rome, Italy; ${ }^{5}$ Azienda Sanitaria di Bologna, Bologna, Italy

Background Worldwide, several million cases of genital warts occur each year. The objective of this study was to estimate the prevalence and the incidence of external genital warts (eGW) in a sample of women attending gynaecological ambulatories and to estimate the number of women with eGW among women aged 15-64 years in Italy 